\title{
Editorial
}

\section{Special issue: Mobile web data analytics (part II)}

\author{
Zili Zhang ${ }^{\text {a,* }}$, Li Liu ${ }^{\mathrm{b}}, \mathrm{Li} \mathrm{Li}^{\mathrm{a}}{ }^{\mathrm{a},}$, and Xiangliang Zhang ${ }^{\mathrm{c}}$ \\ ${ }^{a}$ Southwest University, China \\ E-mails: zili.zhang@deakin.edu.au,lily@swu.edu.cn \\ ${ }^{\mathrm{b}}$ Chongqing University, China \\ ${ }^{\mathrm{c}}$ King Abdullah University of Science and Technology, Saudi Arabia
}

It is essential to constantly collect data with various mobile applications from diverse sources, such as smartphones and ubiquitous sensors. However, how do you conduct the analysis on such a mass of mobile data or mobile web data aiming to solve issues in different areas of applications, including human behavior recognition, medication, recommendation and transportation? Nowadays, research in mobile and social computing environments is turning to novel concepts to address the challenge of data processing and analyzing. This special issue Mobile web data analytics addresses issues of data management in mobile and social computing environments with a special focus on data processing and applications. The goal of this special issue is to build a forum for researchers from academia and industry to investigate challenging and innovative research issues on the subject, which combines data analytics within mobile and social environment and to explore creative concepts, theories, innovative technologies and intelligent solutions. We intend this special issue to act as an initial place where people from different areas can find a forum to discuss issues of data management and processing in new and emerging mobile computing environments.

We accepted 11 papers that provide deep research results to report the advance of mobile web data analytics and applications. These papers are grouped into

\footnotetext{
*Corresponding authors. E-mails: zili.zhang@deakin.edu.au, lily@swu.edu.cn.
}

two special issues. This second special issue contains 6 papers.

In this special issue, the first contribution is "Determining senior wellness status using an intelligent system based on wireless sensor networks and bioinformation" by Wu et al., which proposed a system based on biological data, such as body temperature, heart rate and blood pressure, and activity data of the elderly living in a stable environment, such as a nursing home, to determine their wellness conditions. The second paper "Recognizing diseases from physiological time series data" by Wang et al. recognized diseases from physiological data by unique combinations of representative patterns for different diseases. The third paper "Automatic domain terminology extraction and its evaluation for domain knowledge graph construction" by Luo et al. presented an improved Domain Term Extraction-Improvement (TDE-I) method based on relative co-occurrence rate, which automatically extracted domain basic terminologies and domain compound terminologies. In the fourth paper "Stochastic gradient descent with variance reduction technique", Zhang et al. presented a novel variance reduction technique which applied the moving average of gradient termed SMVRG. The fifth paper "Automatic Chinese character similarity measurement" by Liu et al. presented a machine learning (regression) approach to measure the similarity between two Chinese characters, based on the information which not only included the glyph, but also pronunciation (pinyin) and semantic meaning derived from HowNet. In the sixth paper 
"Speech emotion recognition of Chinese elderly people", Wang et al. proposed a six-layer wavelet packet coefficients model for speech emotion recognition of senior people in China.

In summary, the goal of this special issue is to crystallize the emerging mobile web data technologies and trends into positive efforts to focus on the most promising solutions in applications and optimization for current techniques. The papers show that mobile data technologies are essential for many applications. This special issue aims to look for new opportunities in emerging mobile computing environments.

\section{Biographies}

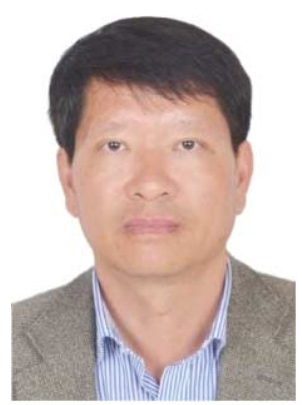

Zili Zhang is a professor at Southwest University, Chongqing, China, and a senior lecturer at the School of Information Technology at Deakin University. He received his BSc from Sichuan University, MEng from Harbin Institute of Technology, and Ph.D. from Deakin University, all in computing. He authored or co-authored over 160 refereed papers in international journals and conference proceedings, one monograph, and seven textbooks. His research interests include bio-inspired artificial intelligence, agent-based computing, and big data analysis. $\mathrm{He}$ is a distinguished member of Chinese Computer Federation (CCF).

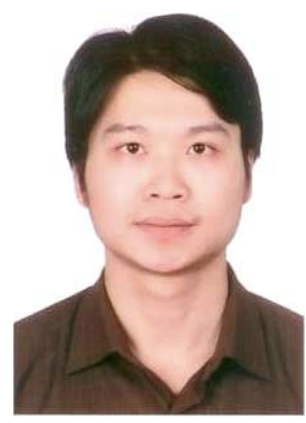

Li Liu is an associate professor at Chongqing University. $\mathrm{He}$ also serves as a Senior Research Fellow of the School of Computing at the National University of Singapore. Liu received his Ph.D. in Computer Science from the Université Paris-sud XI in 2008. He has served as an associate professor at Lanzhou University in China. His research interests include pattern recog- nition, data analysis, and their applications on human behaviors. He aims to contribute to interdisciplinary research of computer science and human related disciplines. Liu has published widely in conferences and journals with over 50 peer-reviewed publications. Liu has been the Principal Investigator of several funded projects from government and industry.

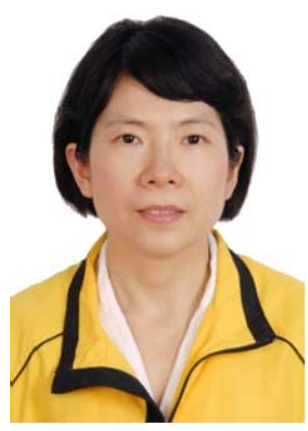

$\mathbf{L i} \mathbf{L i}$ is a professor at the School of Computer and Information Science at Southwest University, Chongqing, China. She received her Ph.D. degree in Computer Science from Swinburne University of Technology, Melbourne, Australia in 2006. Her research interests include data analysis, data mining and machine learning. She has published over 70 peer-reviewed papers. She is a senior member of China Computer Federation and Chinese Association for Artificial Intelligence.

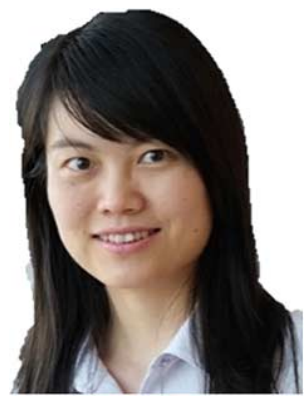

Xiangliang Zhang is an associate professor at King Abdullah University of Science and Technology. She earned her Ph.D. degree in computer science from the INRIAUniversite Paris-Sud, France, in July 2010. She received M.S. and B.S. degrees from Xi'an Jiaotong University, China, in 2006 and 2003, respectively. Her research mainly focuses on developing automated methods for machine learning and data mining to discover and manage knowledge from complex and large-scale data sets for diverse applications. She has authored or coauthored over 40 research papers in referred international journals and conference proceedings. She is the guest editor of Mathematics in Computer Sciences and IEEE Network. She serves as Technical Program Committee member for SIGKDD, ICDE, ICDM, CIKM, etc. 